\section{Cómo interpretar guías de práctica clínica elaboradas con metodología GRADE}

\author{
CAROLINA MENDOZA ${ }^{1, \mathrm{a}, \mathrm{g}}$, PATRICIA KRAEMER ${ }^{1, \mathrm{~b}}$, PALOMA HERRERA $^{1, \mathrm{c}}$, \\ PAMELA BURDILES ${ }^{1, \mathrm{~d}}$, DINO SEPÚLVEDA ${ }^{1,3, \mathrm{f}}$, ELIOZKA NÚÑNZZ $^{1, \mathrm{e}}$, CECI- \\ LIA MUÑOZ ${ }^{1}$, IGNACIO NEUMANN ${ }^{2, g}$
}

\section{Clinical guidelines using the GRADE system (Grading of Recommendations Assessment, Development and Evaluation)}

The Ministry of Health of Chile, aiming to improve the quality of clinical practice guidelines, gradually incorporated the GRADE system (Grading of Recommendations, Assessment, Development and Evaluation) to develop evidence based recommendations. This system summarizes and evaluates the certainty of the available evidence. It moves from evidence to decision in a systematic and transparent manner, based on four main dimensions: balance between benefits and harms, certainty of evidence, patient's values and preferences and use of resources. The GRADE system produces strong and conditional recommendations. Strong recommendations provide confidence that the favorable consequences of an intervention clearly outweigh the adverse consequences, or vice versa. These recommendations apply to a broad range of patients and circumstances. Conditional recommendations, however, indicate that there is a close balance between favorable and unfavorable consequences of the intervention, there is uncertainty in the magnitude of benefits or adverse effects, there is uncertainty or variability in values and preferences of individuals or costs are not justified. These recommendations apply to many patients, but not all of them: ideally they should be discussed with each person. To achieve a better implementation of the recommendations made with GRADE methodology, health professionals should know the meaning of strong and conditional recommendations and they should be able to critically assess of them.

(Rev Med Chile 2017; 145: 1463-1470)

Key words: Chile; Evidence-Based Practice; Practice Guidelines as Topic.

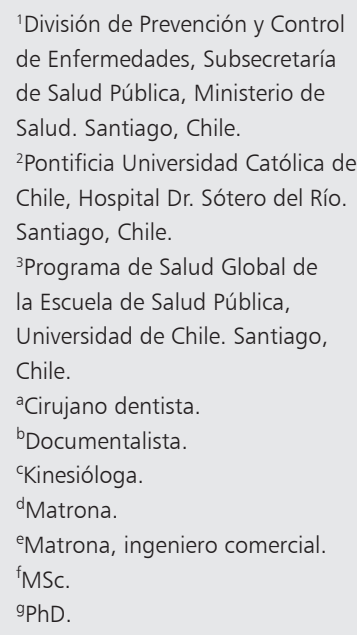

Fuente de apoyo financiero: no se recibió apoyo financiero.

Los autores no declaran conflictos de interés.

Recibido el 8 de mayo de 2017 aceptado el 21 de diciembre 2017

Correspondencia a: Carolina Mendoza Mac Iver 541. Santiago, Chile. cmendoza@minsal.cl
E n Chile, el desarrollo de guías de práctica clínica (GPC) se inició junto con la ley No 19.966 de 2004 que establece un Régimen de Garantías Explícitas en Salud (GES) ${ }^{1}$. Desde ese momento, el Ministerio de Salud ha estado permanentemente elaborando y actualizando guías de práctica clínica para orientar a los profesionales de salud sobre las mejores prácticas para atender los problemas de salud incluidos en el Régimen GES. Sin embargo, en los últimos años ha habido cambios significativos en la forma de elaborar GPC, pasando de métodos informales basados en el consenso de expertos a métodos sistemáticos y transparentes de búsqueda y síntesis de evidencia, seguidos por un proceso estructurado para moverse de la evidencia a la decisión. En sintonía con esta tendencia internacional, el 2014 el Ministerio de Salud incorporó el sistema GRADE en su proceso de elaboración de GPC.

El sistema GRADE (del inglés Grading of Re- 
commendations Assessment, Development and Evaluation $)^{2}$ permite sintetizar la evidencia, evaluar la certeza en esta evidencia (o calidad de la evidencia) e ir de la evidencia a la recomendación de una manera sistemática y transparente ${ }^{3,4}$. Además, permite diferenciar entre recomendaciones destinadas a todos o casi todas las personas y recomendaciones que requieren evaluar individualmente cada situación clínica (Figura 1).

Este artículo tiene el propósito de orientar a los profesionales de salud en la interpretación y uso de las recomendaciones formuladas con el sistema GRADE, incluyendo herramientas para su evaluación crítica.

\section{1. ¿Cómo interpretar una recomendación formulada con sistema GRADE?}

\section{¿Qué significa la fuerza de una recomendación?}

Las recomendaciones pueden ser fuertes o condicionales. Las fuertes indican que el panel tiene alta confianza en que las consecuencias favorables de la intervención superan claramente a las consecuencias desfavorables, si la recomendación es a favor de una intervención; o que las consecuencias desfavorables superan claramente a las favorables, si es en contra. En las GPC elaboradas por el Ministerio de Salud se utiliza el siguiente formato para las recomendaciones fuertes: "el Ministerio de Salud recomienda" o "recomendamos", seguido de "recomendación fuerte".

Las recomendaciones condicionales (o también llamadas débiles) indican que: i. el panel considera que hay un balance cercano entre los beneficios y riesgos de la intervención; ii. existe incertidumbre sobre la magnitud de los beneficios o efectos adversos; iii. existe incertidumbre o variabilidad en los valores y preferencias de las personas; o iv. los costos de la intervención no están justificados en todos los casos. En las GPC desarrolladas por el Ministerio de Salud se usa el siguiente formato para las recomendaciones condicionales: "el Ministerio de Salud sugiere" o "sugerimos", seguido de "recomendación condicional".

\section{¿Qué significa la certeza en la evidencia?}

El sistema GRADE se aplica a conjuntos de evidencia que reflejan los efectos de las alternativas preventivas, diagnósticas o terapéuticas de interés, entendiendo por conjunto de evidencia a los estudios con diseño similar que responden a una misma pregunta clínica. El concepto de certeza en la evidencia se refiere a la confianza que tenemos en que los estimadores del efecto (RR, OR, DM, HR) son apropiados para apoyar una recomendación determinada. El sistema GRADE propone cuatro niveles de certeza en la evidencia: alto, moderado, bajo y muy bajo ${ }^{5}$.

La determinación del nivel de certeza en la evidencia se realiza a través de una evaluación sistemática y transparente del diseño de los estudios incluidos, de las limitaciones de este cuerpo de evidencia (riesgo de sesgo, inconsistencia, imprecisión, evidencia indirecta, sesgo de publicación) o de las circunstancias especiales que pueden aumentar nuestra confianza en los estimadores (gran magnitud del efecto, sesgo residual actuando en dirección contraria, gradiente dosis respuesta $)^{6}$ (Figura 2).

\section{2. ¿Cómo evaluar críticamente las recomenda- ciones formuladas con sistema GRADE?}

\section{¿El panel consideró explícitamente todos los desenlaces relevantes para los pacientes?}

Las GPC responden preguntas clínicas sobre una población, intervención y comparación específicas. Los desenlaces permiten evaluar el balance entre beneficios y riesgos de esa intervención y los usuarios de una GPC deberán evaluar si el grupo elaborador incluyó todos los desenlaces relevantes para los pacientes.

Los desenlaces importantes para los pacientes incluyen generalmente mortalidad, morbilidad (por ejemplo, duración de síntomas, hospitalizaciones, náuseas) y aspectos reportados por las personas (por ejemplo, dolor, calidad de vida). Sin embargo, los desenlaces que no son factibles de ser modificados por la intervención no son relevantes para la toma de decisión y no deberían ser considerados. Por ejemplo, la mortalidad es un desenlace generalmente importante para los pacientes; sin embargo, no es relevante para tomar la decisión sobre recomendar o no el uso de barniz de flúor para la prevención de caries radiculares en adultos de 60 años y más, porque no es plausible que esta intervención afecte el riesgo de morir ${ }^{7}$.

Los desenlaces sustitutos o intermedios tienen una relación variable con los desenlaces importantes para las personas, pero nunca son importantes por sí mismos. Por ejemplo, la hipertensión arte- 


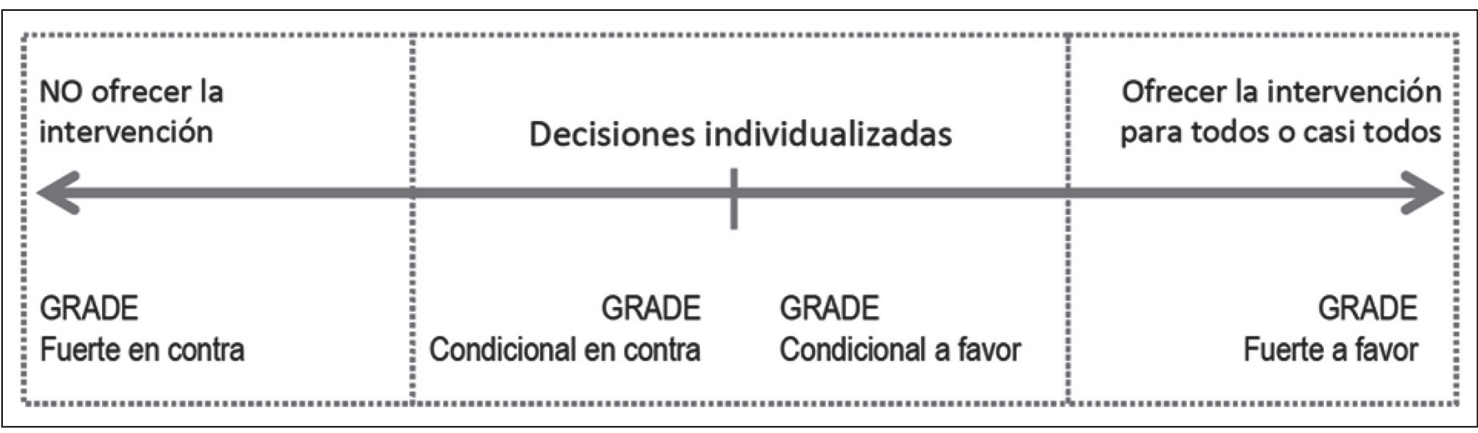

Figura 1. Dirección y fuerza de las recomendaciones según el sistema GRADE.

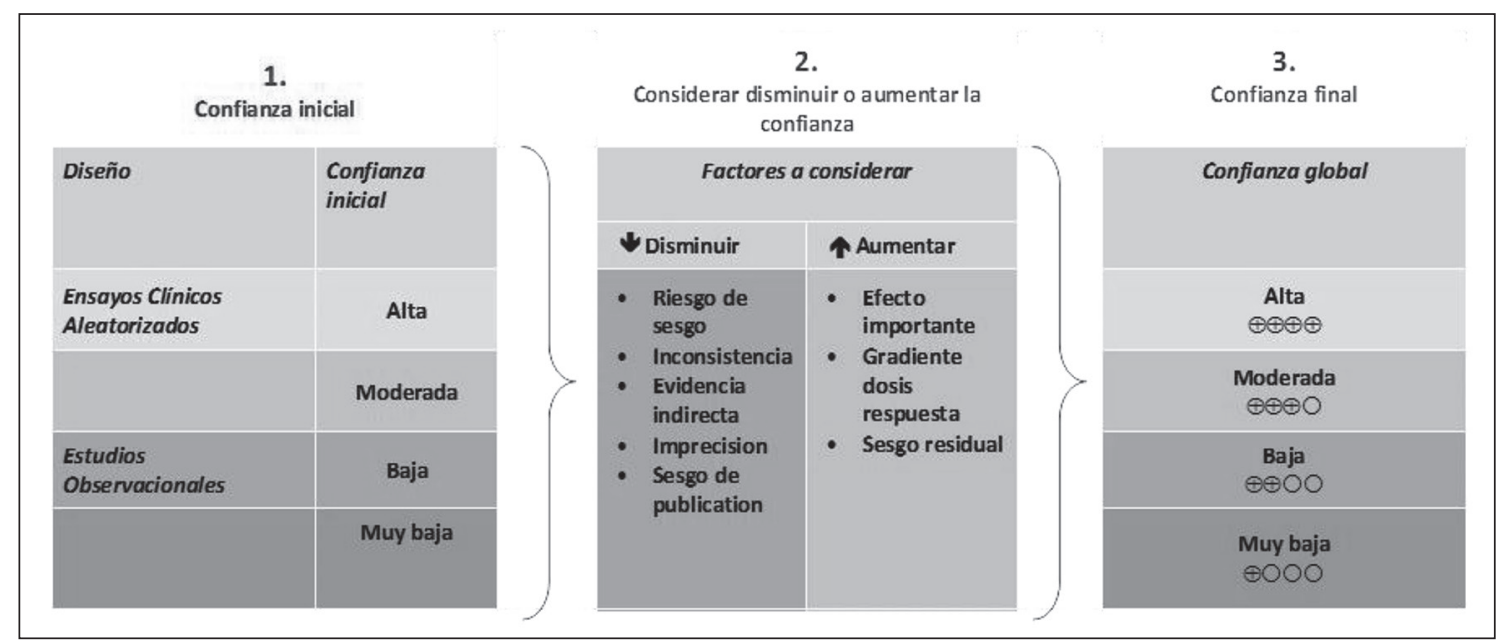

Figura 2. Sistema GRADE para evaluar la certeza en la evidencia para cada desenlace considerando el conjunto de estudios. * Los criterios para aumentar la calidad de la evidencia se utilizan por lo general sólo cuando se tiene evidencia de estudios observacionales.

rial es un factor de riesgo para el desarrollo de daño vascular que se traduce en un aumento de eventos cardiovasculares adversos como infarto agudo al miocardio, accidente vascular encefálico, insuficiencia cardiaca y mortalidad cardiovascular. Por lo tanto, podríamos inferir que cualquier droga hipotensora podría tener un efecto en la reducción de los eventos cardiovasculares adversos mencionados. Sin embargo, el atenolol reduce la presión arterial, pero no parece prevenir la aparición de infarto, accidente vascular encefálico o mortalidad cardiovascular en pacientes con hipertensión arterial esencial. En este ejemplo, la presión arterial es un desenlace intermedio y el infarto agudo al miocardio y la mortalidad cardiovascular son desenlaces importantes para los pacientes. Tomar decisiones basadas en beneficios en los desenlaces intermedios no necesariamente genera beneficios en los desenlaces importantes para los pacientes ${ }^{8}$.

\section{¿La recomendación fue basada en evidencia actualizada?}

El grupo elaborador debe basar sus estimaciones de riesgos y beneficios de una intervención a partir de revisiones sistemáticas. Cuando no existen revisiones actualizadas o de buena calidad metodológica, el grupo elaborador puede hacer su propia revisión sistemática. En ambos casos es importante describir los procesos de búsqueda y síntesis de evidencia realizados. Los profesionales de salud usuarios de una guía de práctica clínica tendrán que juzgar si los métodos usados 
en la revisión sistemática fueron suficientemente rigurosos ${ }^{9}$.

Las recomendaciones que no han sido formuladas utilizando evidencia actualizada pueden promover intervenciones inefectivas o dañinas, por lo que la actualización permanente de las guías clínicas es fundamental, especialmente en áreas donde hay un gran desarrollo de la investigación clínica. Por ejemplo, una proporción importante de la evidencia disponible sobre efectividad del oseltamivir en personas con influenza fue dada a conocer recién en el año 2014 tras gran presión internacional sobre el fabricante ${ }^{10}$. Según esta nueva evidencia, oseltamivir probablemente acorta unas horas la duración de los síntomas, pero no tiene un efecto claro en reducir las complicaciones $\mathrm{u}$ hospitalizaciones por influenza ${ }^{11}$. Considerando esta nueva evidencia, la versión 2015 de la GPC sobre el uso de oseltamivir en personas con diagnóstico o sospecha de influenza del Ministerio de Salud sugirió no usar oseltamivir en adultos o niños de bajo riesgo, y solo considerar su uso en pacientes con mayor riesgo de complicación o en que las consecuencias de una complicación pueden ser graves ${ }^{12}$.

\section{¿La fuerza de la recomendación es la apropiada?}

La fuerza de una recomendación tiene implicancias directas sobre su implementación y las recomendaciones inapropiadamente definidas como fuertes pueden entregar mensajes erróneos a los profesionales de salud.

Cuando la certeza en la evidencia es moderada o alta, los profesionales de salud pueden estar confiados en que la evidencia es cré́ble. En estas circunstancias, la evidencia justifica una recomendación fuerte cuando además el balance entre consecuencias favorables y desfavorables no es cercano (las consecuencias favorables claramente superan las desfavorables, o viceversa), hay confianza y poca variabilidad en los valores y preferencias de los pacientes y los costos de la implementación de la recomendación están justificados en todos o casi todos los casos.

Cuando la certeza en la evidencia es baja o muy baja, hay mucha incertidumbre respecto de los efectos de la intervención, por lo tanto debiéramos esperar una recomendación condicional. Si bien existen circunstancias en que el panel pudiera formular recomendaciones fuertes basadas en baja o muy baja certeza en la evidencia, estas circunstancias son excepcionales. Un estudio que evalúo 33 GPC desarrolladas por la Organización Mundial de la Salud entre 2007 y 2012 mostró que de 456 recomendaciones formuladas con sistema GRADE, 160 eran recomendaciones fuertes con baja o muy baja certeza en la evidencia. De ellas, solo $16 \%$ estaba correctamente justificada ${ }^{13}$. Esto muestra la necesidad de evaluar cuidadosamente las recomendaciones fuertes basadas en baja o muy baja certeza en la evidencia.

La variabilidad en los valores y preferencias de los pacientes es otro factor clave a considerar para evaluar la fuerza de la recomendación. En situaciones de baja o muy baja certeza en la evidencia, la actitud de los pacientes frente a beneficios o daños inciertos puede hacer cambiar el balance entre las consecuencias favorables y desfavorables. Por ejemplo, en la GPC "Prevención de mucositis oral en personas con cáncer" desarrollada por el Ministerio de Salud en 2014, el panel formuló una recomendación condicional a favor del uso de crioterapia oral durante la administración de quimioterapia en personas con cáncer (recomendación condicional; certeza en la evidencia baja). En este caso, los efectos indeseados son pequeños, los costos bajos, pero los beneficios son inciertos. En este escenario, algunos pacientes podrían elegir usar crioterapia para obtener un beneficio incierto, pero otros preferirían no usarlo, valorando más las posibles molestias asociadas al uso de hielo en la boca ${ }^{14}$. Dada la incertidumbre respecto de los beneficios de la crioterapia y las distintas actitudes que las personas pueden tener frente a la incertidumbre, una recomendación condicional es adecuada.

\section{¿La recomendación es clara y orientada a la acción?}

Las recomendaciones formuladas con sistema GRADE deben incluir el nivel de certeza en la evidencia y la fuerza de la recomendación. La redacción debe ser estandarizada y detallar claramente a quien está dirigida, cuál es la acción, en qué circunstancia debe aplicarse y la alternativa con la que se compara la acción. Las recomendaciones con lenguaje vago o que solo entregan un resumen de la evidencia no son útiles, ya que quedan abiertas a la interpretación de cada persona que las use, lo que dificulta su implementación. Por ejemplo, la versión 2014 de la guía sobre el manejo de pacientes con influenza del Ministerio 
de Salud incluyó la siguiente recomendación: "La indicación de antivirales debe realizarse evaluando en base a la información clínica de cada caso, balanceando riesgos vs beneficios considerando la escasa evidencia de su efectividad y efectos secundarios. En adultos y niños con factores de riesgo para enfermedad respiratoria aguda grave cuyo cuadro clínico corresponda a un caso sospechoso o confirmado de influenza se recomendaría tratamiento antiviral antes de $48 \mathrm{~h}$ desde la aparición de los síntomas" ${ }^{15}$. En esta recomendación la acción -indicar antivirales- queda oculta dentro de un texto extenso y vago. Aún más importante, no queda claro qué hacer con las personas sin factores de riesgo. Implícitamente se sugiere no usar antivirales, pero queda abierto a la interpretación del lector. La versión 2015 sobre el uso de oseltamivir en personas con diagnóstico o sospecha de influenza reemplazó esta recomendación por recomendaciones breves y orientadas a la acción: 1. En adultos de bajo riesgo con sospecha o diagnóstico de influenza, el Ministerio de Salud sugiere NO USAR oseltamivir (recomendación condicional, certeza en la evidencia baja); 2. En adultos de alto riesgo con sospecha o diagnóstico de influenza, el Ministerio de Salud sugiere USAR oseltamivir (recomendación condicional, certeza en la evidencia baja) ${ }^{12}$.

\section{¿La recomendación entrega suficiente información para su implementación?}

Las recomendaciones, especialmente las condicionales, deben entregar explícitamente la información necesaria para su implementación. Esto contribuye a aumentar la transparencia del proceso de formulación de recomendaciones y facilita la toma de decisión compartida con el paciente.

La información sobre dosificación, vía de administración, frecuencia y duración del uso de la intervención resulta fundamental para la implementación. Puede estar contendida en los comentarios que acompañan la recomendación o en otras secciones de la guía. Por ejemplo, en la guía clínica "Uso ambulatorio de oseltamivir para personas con sospecha o diagnóstico de influenza", la recomendación sobre uso de oseltamivir en adultos de alto riesgo con sospecha o diagnóstico de influenza incluye la dosificación, frecuencia y duración del tratamiento con oseltamivir ( $75 \mathrm{mg}$ cada $12 \mathrm{~h}$ por 5 días) ${ }^{12}$.
Las tablas "resumen de la evidencia" o tablas SoF por sus siglas en inglés (Summary of Findings) ${ }^{3}$ entregan información sobre los estimadores de efectos (efectos relativos y absolutos) y la certeza en la evidencia para cada uno de los desenlaces importantes para los pacientes. Esta información puede ser útil para apoyar la toma de decisión compartida entre el profesional de salud y el paciente. La Tabla 1 corresponde al resumen de evidencia para el uso de oseltamivir en adultos con sospecha o diagnóstico de influenza.

\section{3. ¿Cómo usar las recomendaciones formuladas con sistema GRADE?}

\section{Recomendaciones fuertes}

Si las recomendaciones fuertes están justificadas (de acuerdo a lo discutido en la sección anterior), los profesionales de salud pueden aplicar la recomendación a todos o casi todos los pacientes y en todos o casi todas las circunstancias, sin necesidad de revisar la evidencia que apoya esta recomendación.

Por ejemplo, la GPC "Salud oral integral para adultos de 60 años: Prevención y tratamiento de caries radiculares" desarrollada por el Ministerio de Salud en 2015 recomienda el uso de pastas dentales con 5.000 ppm de flúor en lugar de pastas dentales convencionales (con 1.000-1.500 ppm de flúor) en adultos de 60 años y más con lesiones de caries radiculares (recomendación fuerte, certeza en la evidencia moderada $)^{7}$. En este caso, la certeza en la evidencia es moderada (el nivel de certeza en la evidencia más bajo entre los desenlaces críticos corresponde al desenlace inactivación de lesiones de caries radiculares y es moderado debido a inconsistencia entre los estudios), el balance entre riesgos y beneficios favorece claramente a los beneficios, los costos son pequeños y están justificados y la mayoría de las personas aceptaría usar la pasta de 5.000 ppm. En consecuencia, el panel consideró que todas o casi todas las personas adecuadamente informadas elegirían usar pastas con 5.000 ppm de flúor en lugar de pastas convencionales. Por lo tanto, una discusión detallada con el paciente sobre los riesgos y beneficios de la intervención no es necesaria. A pesar de lo anterior, siempre pueden existir circunstancias excepcionales en que los profesionales de salud o pacientes no adhieran a una recomendación fuerte. Por ejemplo, sería 


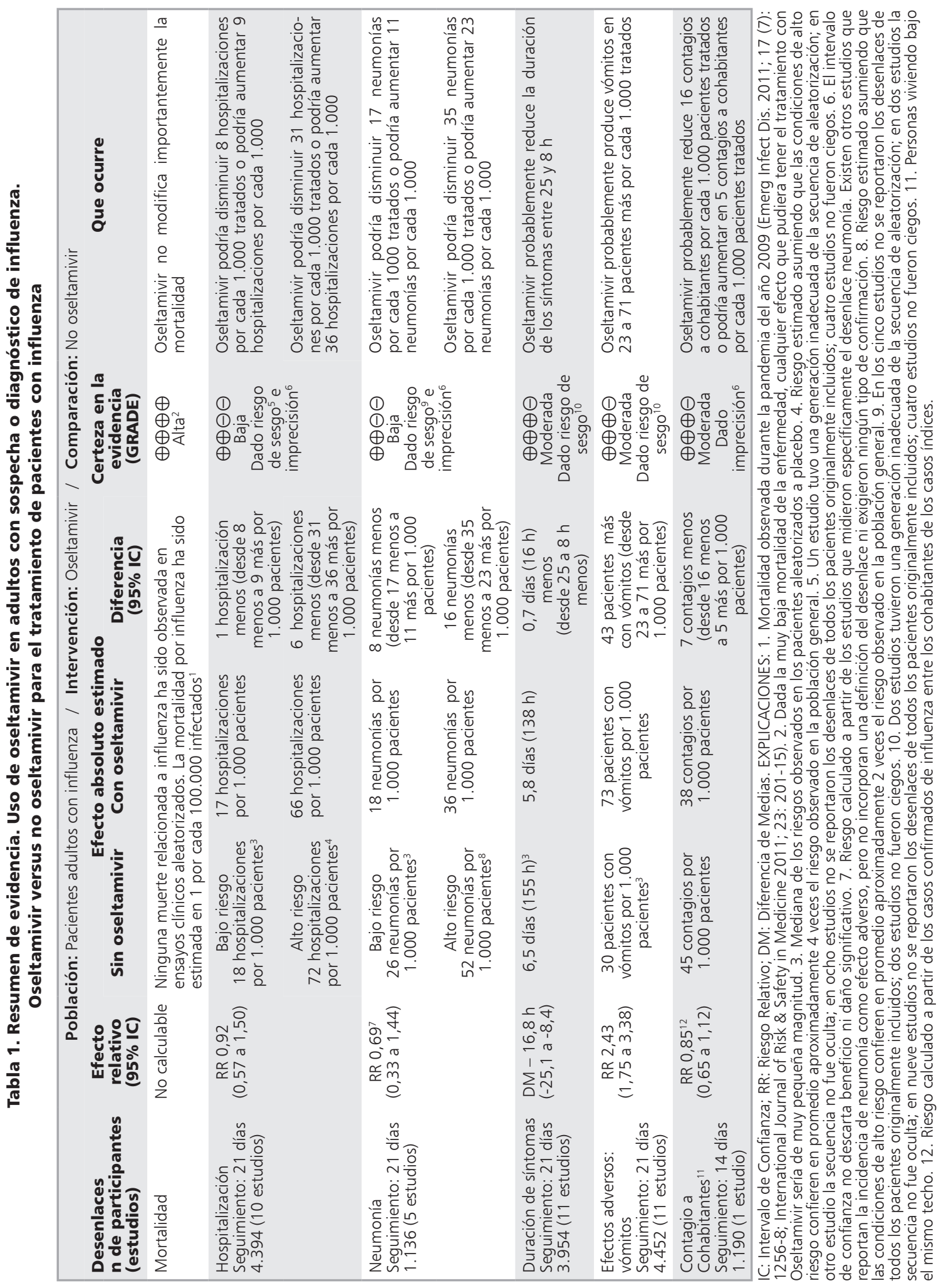


un error indicar pastas dentales con flúor a una persona alérgica a alguno de los componentes de las pastas de diente. Afortunadamente, estas circunstancias son muy inusuales.

\section{Recomendaciones condicionales}

Al utilizar el sistema GRADE, haciendo una evaluación crítica de la evidencia y considerando los valores y preferencias de los pacientes, la gran mayoría de las recomendaciones son condicionales, incluso en áreas clínicas en que hay gran desarrollo de ensayos clínicos y revisiones sistemáticas. Se estima que cerca de dos tercios de todas la recomendaciones elaboradas con sistema GRADE son condicionales.

Las recomendaciones condicionales pueden ser aplicadas a la mayoría de las personas, pero no a todas. Idealmente, los profesionales de salud deben familiarizarse con la evidencia que apoya la recomendación y considerar los factores que la hacen condicional (por ejemplo, el balance cercano entre riesgos y beneficios o la variabilidad en valores y preferencias de las personas). En estos casos, una toma de decisión compartida entre el profesional de salud y el paciente es la conducta más adecuada a seguir. Es importante discutir con el paciente los potenciales beneficios y riesgos de las alternativas, para tomar la mejor decisión considerando la evidencia disponible y sus valores y preferencias.

Por ejemplo, en la GPC "Uso ambulatorio de oseltamivir para personas con sospecha o diagnóstico de influenza" se sugiere no usar oseltamivir en adultos de bajo riesgo con sospecha o diagnóstico de influenza (recomendación condicional, certeza en la evidencia baja $)^{12}$. Esta recomendación es condicional debido a que existe considerable incertidumbre respecto de los beneficios de oseltamivir (certeza en la evidencia baja en los desenlaces hospitalización y neumonía), el balance entre las consecuencias favorables y desfavorables es cercano, sus costos son altos y existe incertidumbre respecto de los valores y preferencias de los pacientes.

La tabla resumen de evidencia presenta información que puede ayudar a tomar una decisión compartida con los pacientes. Los efectos absolutos estimados permiten entregar información sobre las consecuencias esperadas de las alternativas disponibles en un lenguaje relativamente simple. Por ejemplo, en el caso de los adultos de bajo riesgo con diagnóstico o sospecha de influenza, el profesional de salud podría explicar al paciente que en relación a los beneficios del uso de oseltamivir, existe bastante incertidumbre sobre el efecto del oseltamivir en la reducción de las hospitalizaciones (podría reducir 8 o aumentar 9 hospitalizaciones por cada 1.000 pacientes tratados). Por otro lado, tenemos más certeza en los efectos sobre la reducción de la duración de síntomas (oseltamivir probablemente reduce el período sintomático en $16 \mathrm{~h}$ ). En cuanto a los riesgos, sabemos que el uso de oseltamivir probablemente produce vómitos en 43 personas más por cada 1.000 tratados (desde 23 a 71 personas más con vómitos) (Tabla 1). Dependiendo de la importancia que el paciente asigne a cada uno de estos desenlaces y del balance que realice entre las consecuencias favorables y desfavorables (incluyendo los costos), tomará la decisión que mejor se ajuste a sus preferencias. Ambas decisiones - usar o no usar oseltamivir-son razonables y están dentro de lo que una recomendación condicional expresa.

\section{Conclusiones}

Este artículo corresponde a una guía para interpretar, evaluar críticamente y utilizar adecuadamente las recomendaciones elaboradas con sistema GRADE, haciendo énfasis en las diferencias entre recomendaciones fuertes $y$ condicionales. Se presentan ejemplos reales de recomendaciones incluidas en las guías de práctica clínica desarrolladas por el Ministerio de Salud. Esperamos que este material sea de utilidad para los profesionales de salud quienes deben implementar las recomendaciones en distintos escenario de la práctica clínica.

\section{Referencias}

1. Ministerio de Salud. Ley No 19.966. Establece un régimen de garantías en salud [Internet]. 2004. Disponible en: http://bcn.cl/1m68a [Consultado el 11 de octubre de 2016].

2. Guyatt GH, Oxman AD, Schünemann HJ, Tugwell P, Knottnerus A. GRADE guidelines: a new series of articles in the Journal of Clinical Epidemiology. J Clin Epidemiol 2011; 64 (4): 380-2.

3. Guyatt G, Oxman AD, Akl EA, Kunz R, Vist G, Brozek J, et al. GRADE guidelines: 1. Introduction-GRADE 
evidence profiles and summary of findings tables. J Clin Epidemiol 2011; 64 (4): 383-94.

4. Alonso-Coello P, Oxman AD, Moberg J, Brignardello-Petersen R, Akl EA, Davoli M, et al. GRADE Evidence to Decision (EtD) frameworks: a systematic and transparent approach to making well informed healthcare choices. 2: Clinical practice guidelines. BMJ 2016; 353: i2089.

5. Hultcrantz M, Rind D, Akl EA, Treweek S, Mustafa RA, Iorio A, et al. The GRADE Working Group clarifies the construct of certainty of evidence. J Clin Epidemiol 2017; 87: 4-13.

6. Balshem $H$, Helfand $M$, Schünemann HJ, Oxman AD, Kunz R, Brozek J, et al. GRADE guidelines: 3. Rating the quality of evidence. J Clin Epidemiol 2011; 64 (4): 401-6.

7. Ministerio de Salud. Guía clínica AUGE. Salud oral integral para adultos de 60 años. Prevención y tratamiento de caries radiculares [Internet]. 2015. Disponible en: http://web.minsal.cl/wp-content/uploads/2015/10/ Salud_Oral60anios.pdf [Consultado el 11 de octubre de 2016].

8. Neumann I, Claro JC. Outcomes intermedios. Rev Med Chile 2012; 140 (1): 113-6.

9. Murad MH, Montori VM, Ioannidis JPA, Jaeschke R, Devereaux PJ, Prasad K, et al. How to read a systematic review and meta-analysis and apply the results to patient care: users' guides to the medical literature. JAMA 2014; 312 (2): 171-9.

10. The BMJ. Tamiflu campaign [Internet]. Disponible en: http://www.bmj.com/tamiflu [Consultado el 11 de octubre de 2016].

11. Jefferson T, Jones M, Doshi P, Del Mar C, Hama R. Neuraminidase inhibitors for preventing and treating influenza in adults and children. Cochrane Database Syst Rev 2014; 4 :CD008965.

12. Ministerio de Salud. Guía de práctica clínica. Uso ambulatorio de oseltamivir para personas con sospecha o diagnóstico de influenza [Internet]. 2015. Disponible en: http://web.minsal.cl/sites/default/files/ files/GUIA\%20DE\%20PRACTICA\%20CLINICA_INFLUENZA_2015_07_21.pdf [Consultado el 11 de octubre de 2016].

13. Alexander PE, Brito JP, Neumann I, Gionfriddo MR, Bero L, Djulbegovic B, et al. World Health Organization strong recommendations based on low-quality evidence (study quality) are frequent and often inconsistent with GRADE guidance. J Clin Epidemiol 2016; 72: 98-106.

14. Ministerio de Salud. Guía clínica. Prevención de mucositis oral en personas con cáncer [Internet]. 2014 Disponible en: http://web.minsal.cl/sites/default/files/files/ GPC\%20manejo\%20lesiones\%20mucosa\%20oral_\%20 dic\%202014\%20(2).pdf [Consultado el 11 de octubre de 2016].

15. Ministerio de Salud. Guía de práctica clínica. Prevención, diagnóstico y manejo clínico de casos de influenza [Internet]. Santiago, Chile: Ministerio de Salud; 2014. Disponible en: http://web.minsal.cl/sites/default/files/ files/GUIA\%20CLINICA\%20INFLUENZA\%202014_ imprimir.pdf [Consultado el 11 de octubre de 2016]. 